\title{
Targeted Nanoparticles for Cancer Therapy: Promises and Challenges
} Kytai Truong Nguyen

Department of Bioengineering, University of Texas at Arlington, Arlington, TX, USA

\section{Introduction}

Cancer, a disease characterized by the uncontrolled growth and spread of abnormal cells, is still the second most common cause of death in the U.S. According to the American Cancer Society, about 571,950 Americans are expected to die in 2011 due to cancer, and that means more than 1,500 deaths per day. Current treatments for various cancers include surgery, radiation, hormone therapy, and chemotherapy. Although these conventional therapies have improved patients' survival, they also have several limitations. For example, conventional cancer chemotherapy has the cancer therapeutic agents distributing non-specifically in the human body, thus these drugs affect both cancerous and normal cells. This non-specific distribution of drugs limits the therapeutic dose within cancer cells while providing excessive toxicities to normal cells, tissues, and organs; and thereby causing several adverse side effects including hairloss, weakness, and organ dysfunction, leading to a low quality of life for cancer patients.

Nanoparticles (NPs) have been of significant interest over the last decade as they offer great benefits for drug delivery to overcome limitations in conventional chemotherapy [1-3]. They can not only be formed in a range of sizes $(1-1000 \mathrm{~nm})$ but also be made using a variety of materials including polymers (e.g. biodegradable polymeric nanoparticles, dendrimers), lipids (e.g. solid-lipid nanoparticles, liposomes), inorganic materials (e.g. metal nanoparticles, quantum dots), and biological materials (e.g. viral nanoparticles, albumin nanoparticles). In addition, they can be tailored to simultaneously carry both drugs and imaging probes and designed to specifically target molecules of diseased tissues. Nanoparticles for anti-cancer drug delivery had reached the first clinical trial in the mid-1980s, and the first nanoparticles (e.g. liposomal with encapsulated doxorubicin) had entered the pharmaceutical market in 1995. Since then, numerous new nanoparticles for cancer drug delivery have been approved and/ or are currently under development due to their many advantages. Their advantages include enhancing solubility of hydrophobic drugs, prolonging circulation time, minimizing non-specific uptake, preventing undesirable off-target and side effects, improving intracellular penetration, and allowing for specific cancer-targeting.

\section{Promises of targeted NPs for cancer therapy}

Using targeted nanoparticles to deliver chemotherapeutic agents in cancer therapy offers many advantages to improve drug/gene delivery and to overcome many problems associated with conventional chemotherapy [3-5]. For example, nanoparticles via either passive targeting or active targeting have been shown to enhance the intracellular concentration of drugs/genes in cancer cells while avoiding toxicity in normal cells. In addition, the targeted nanoparticles can also be designed as either $\mathrm{pH}$-sensitive or temperature-sensitive carriers. The $\mathrm{pH}$-sensitive drug delivery system can deliver and release drugs within the more acidic microenvironment of the cancer cells and/or components within cancer cells. The temperature-sensitive system can carry and release drugs with changes in temperature locally in the tumor region provided by sources such as magnetic fields, ultrasound waves, and so on so that combined therapy such as chemotherapy and hyperthermia can be applied. The targeting of nanoparticles to tumors via cancer-specific features/moieties has also been shown to minimize the effects of composition, size, and molecular mass of nanoparticles on their efficacy [6]. Targeted nanoparticles can be further modified or functionalized to reduce toxicity. For example, modifying nanoparticles surface chemistry could reduce their toxicity and immunotoxicity [1].

\section{Challenges of targeted NPs for cancer therapy}

Although targeted nanoparticles have emerged as one strategy to overcome the lack of specificity of conventional chemotherapy, there are also potential risks and challenges associated with this novel strategy. For instance, some cancer cell types would develop drug resistance over the drug treatment course, thereby rendering drugs released from the targeted nanoparticles to be ineffective. Combined therapies, such as the use of targeted nanoparticles for delivering both chemotherapeutics and gene therapeutics, might be effectively delivered and specifically targeted to cancer cells and tissues to overcome this drug resistance and to stop the tumor growth. Another strategy to overcome this drug resistance is to develop multifunctional targeted nanoparticles.

Similar to other new technologies, targeted NPs for cancer therapy also face many challenges. One challenge of targeted NPs is that NPs might change the stability, solubility, and pharmacokinetic properties of the carried drugs. However, these aspects have not been extensively investigated. The shelf life, aggregation, leakage, and toxicity of materials used to make nanoparticles are other limitations for their use. Some materials used to make NPs such aspoly(lactic-co-glycolic acid) (PLGA) have low toxicity, but degrade quickly and do not circulate in tissues long enough for sustained drug/gene delivery. On the other hand, other materials such as carbon nanotubes and quantum dots are durable and can persist in the body for weeks, months, or even years, making them potentially toxic and limiting their use for repeated treatments [7]. New materials to make targeted nanoparticles such as silicon/silica (solid, porous, and hollow silicon nanoparticles) have been developed; however, their use for drug delivery to cancer patients has taken off slowly due to the potential health risks associated with introducing new materials in the human body.

Besides developing new materials and selecting appropriate materials for each specific treatment, other factors need to be optimally selected in order to design better targeted nanoparticles. These factors include the particles size, shape, sedimentation, drug encapsulation efficacy, desired drug release profiles, distribution in the body, circulation, and cost. For instance, in the case of particle size, it has been

Corresponding author: Kytai Truong Nguyen, Assistant Professor, Department of Bioengineering, University of Texas at Arlington, Arlington, TX, USA. Tel: 817272 2540; Fax: 817272-2251; E-mail: knguyen@uta.edu

Received September 15, 2011; Accepted September 17, 2011; Published September 19, 2011

Citation: Nguyen KT (2011) Targeted Nanoparticles for Cancer Therapy: Promises and Challenges. J Nanomedic Nanotechnol 2:103e. doi:10.4172/21577439.1000103e

Copyright: (c) 2011 Nguyen KT. This is an open-access article distributed under the terms of the Creative Commons Attribution License, which permits unrestricted use, distribution, and reproduction in any medium, provided the original author and source are credited. 
Citation: Nguyen KT (2011) Targeted Nanoparticles for Cancer Therapy: Promises and Challenges. J Nanomedic Nanotechnol 2:103e. doi:10.4172/2157-7439.1000103e

Page 2 of 2

well-known that the clearance rate of very small nanoparticles might be high, and most of these nanoparticles might end up in the liver and spleen, thus making the use of targeted nanoparticles impractical and ineffective. On the other hand, larger nanoparticles might be too big to go through small capillaries for drug delivery. Thus selecting the right materials and particle size is another important aspect in targeted NPs for cancer therapy.

Despite extensive research efforts to develop new targeted nanoparticles, only a few of them are in clinical use including Abraxane $^{\circledR}$, Doxil ${ }^{\circledR}$, and Myocet ${ }^{\mathrm{TM}}$ that are approved by FDA. A major account forthe slow development of effective targeted nanoparticles has been due to the lack of knowledge about the distribution and location of targeted nanoparticles after either oral administration or injection. For example, most studies have not examined the targeting efficiency of nanoparticles real time in vivo, thus precise bio-distribution and subsequently therapeutic effects are not well-known. Therefore, detecting cancer (malignant) cells in the body and monitoring treatment effects on these cells in real time is another challenge needed to be overcome to develop efficient targeted nanoparticles.

\section{Conclusion}

Nevertheless, targeted nanoparticles have provided an effective platform for a better and more specific delivery of cancer therapeutics. With our care, desire, and hard work with the patients' benefits in mind to identify both risks and benefits of targeted nanoparticles for cancer therapy, multifunctional targeted nanoparticles can eventually be designed. These targeted nanoparticles would be able to detect cancer cells, visualize their location in the body, deliver drugs to these cells only, circumvent drug resistance, kill cancer cells while sparing normal cells with minimal side effects, monitor treatment effects in real time, and provide feedback whether the patients respond well to the treatments to stop the treatment in time. The role and scope of targeted nanoparticles for drug delivery in cancer therapy is growing, and the development of effective multifunctional targeted nanoparticles will not be far in the future.

\section{References}

1. Subbiah R, Veerapandian M, Yun KS (2010) Nanoparticles: functionalization and multifunctional applications in biomedical sciences. Curr Med Chem 17: 4559-4577.

2. Yoo JW, Chambers E, Mitragotri S (2010) Factors that control the circulation time of nanoparticles in blood: challenges, solutions and future prospects. Curr Pharm Des 16: 2298-2307.

3. Koo H, Huh MS, Sun IC, Yuk SH, Choi K, et al. (2011) In Vivo Targeted Delivery of Nanoparticles for Theranosis. Acc Chem Res.

4. Ali I, Rahis U, Salim K, Rather M A, Wani WA, et al. (2011) Advances in nano drugs for cancer chemotherapy. Curr Cancer Drug Targets 11: 135-46.

5. Heidel JD, Davis ME (2011) Clinical developments in nanotechnology for cancer therapy. Pharm Res 28: 187-99.

6. Saad M, Garbuzenko OB, Ber E, Chandna P, Khandare JJ (2008) Receptor targeted polymers, dendrimers, liposomes: which nanocarrier is the most efficient for tumor-specific treatment and imaging? J Control Release 130: 10714.

7. Jain AK, Das M, Swarnakar NK, Jain S (2011) Engineered PLGA nanoparticles: an emerging delivery tool in cancer therapeutics. Crit Rev Ther Drug Carrier Syst 28: 1-45. 